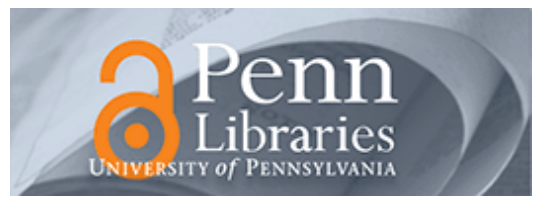

University of Pennsylvania

ScholarlyCommons

Finance Papers

Wharton Faculty Research

9-2016

\title{
Asymmetric Information and Intermediation Chains
}

Vincent Glode

University of Pennsylvania

Christian C. Opp

University of Pennsylvania

Follow this and additional works at: https://repository.upenn.edu/fnce_papers

Part of the Finance Commons, and the Finance and Financial Management Commons

\section{Recommended Citation}

Glode, V., \& Opp, C. C. (2016). Asymmetric Information and Intermediation Chains. American Economic Review, 106 (9), 2699-2721. http://dx.doi.org/10.1257/aer.20140662

This paper is posted at ScholarlyCommons. https://repository.upenn.edu/fnce_papers/235

For more information, please contact repository@pobox.upenn.edu. 


\title{
Asymmetric Information and Intermediation Chains
}

\author{
Abstract \\ We propose a parsimonious model of bilateral trade under asymmetric information to shed light on the \\ prevalence of intermediation chains that stand between buyers and sellers in many decentralized \\ markets. Our model features a classic problem in economics where an agent uses his market power to \\ inefficiently screen a privately informed counterparty. Paradoxically, involving moderately informed \\ intermediaries also endowed with market power can improve trade efficiency. Long intermediation chains \\ in which each trader's information set is similar to those of his direct counterparties limit traders' \\ incentives to post prices that reduce trade volume and jeopardize gains to trade.

\section{Disciplines} \\ Economics | Finance | Finance and Financial Management
}




\title{
Asymmetric Information and Intermediation Chains
}

\author{
By Vincent Glode and Christian OpP*
}

\begin{abstract}
We propose a parsimonious model of bilateral trade under asymmetric information to shed light on the prevalence of intermediation chains that stand between buyers and sellers in many decentralized markets. Our model features a classic problem in economics where an agent uses his market power to inefficiently screen a privately informed counterparty. Paradoxically, involving moderately informed intermediaries also endowed with market power can improve trade efficiency. Long intermediation chains in which each trader's information set is similar to those of his direct counterparties limit traders' incentives to post prices that reduce trade volume and jeopardize gains to trade. (JEL D42, D82, D85, L12, L14)
\end{abstract}

Transactions in decentralized markets often feature the successive involvement of multiple intermediaries. In this paper, we propose a parsimonious model of bilateral trade to study the implications of these types of arrangements in the presence of asymmetric information. Our model initially considers two asymmetrically informed agents who wish to trade an asset in order to realize gains to trade. One agent has market power in pricing the asset, whereas his counterparty is privately informed about the value of the asset. A standard result in models like ours is that trade breaks down with positive probability when the potential

\footnotetext{
* Glode: The Wharton School, University of Pennsylvania, 3620 Locust Walk, Suite 2300, Philadelphia, PA 19104 (e-mail: vglode@wharton.upenn.edu); Opp: The Wharton School, University of Pennsylvania, 3620 Locust Walk, Suite 2300, Philadelphia, PA 19104 (e-mail: opp@wharton.upenn.edu). This paper previously circulated under the title "Adverse Selection and Intermediation Chains." For helpful comments, we thank five anonymous referees, Andy Abel, Franklin Allen, Daniel Andrei, Jim Angel, Ana Babus, Patrick Bolton, Adam Clark-Joseph, Adrian Corum, Shaun Davies, Marco Di Maggio, Xavier Gabaix, Simon Gervais, Michael Gofman, Rick Green, Joseph Harrington, Burton Hollifield, Mark Jenkins, Don Keim, Rich Kihlstrom, Pablo Kurlat, Pete Kyle, Doron Levit, Richard Lowery, Semyon Malamud, Katya Malinova, Asaf Manela, Robert Marquez, Adrian Masters, Bob McDonald, David Musto, Artem Neklyudov, Greg Nini, Martin Oehmke, Maureen O'Hara, Guillermo Ordoñez, Cecilia Parlatore Sirrito, Christine Parlour, David Scharfstein, Norman Schürhoff, Krista Schwarz, Alp Simsek, Andy Skrzypacz, Matt Spiegel, Ilya Strebulaev, Victoria Vanasco, Vish Viswanathan, Brian Weller, Bilge Yilmaz, and seminar participants at Colorado, Columbia, Duke, Federal Reserve Bank of Boston, Lausanne, Michigan, Northwestern, Ohio State, Rochester, Toronto, UC-Berkeley, UC-Irvine, UNC-Chapel Hill, Wharton, AEA meetings, ASU Winter Finance conference, Finance and Economic Networks conference at Wisconsin-Madison, FTG workshop at Chicago-Booth, IDC-Herzliya conference, Money, Banking and Asset Markets conference at Wisconsin-Madison, NFA meetings, NY Fed/NYU-Stern conference on Financial Intermediation, NYU-Stern Microstructure conference, Penn State/Cornell Macro workshop, SED meetings, SFS cavalcade, SITE workshop on New Models of Financial Markets, WFA meetings, and the Wharton conference on Liquidity and Financial Crises. Deeksha Gupta, Michael Lee, and Ruslan Sverchkov provided excellent research assistance. The authors acknowledge financial support from the Cynthia and Bennett Golub Endowed Faculty Scholar award. The authors declare that they have no relevant or material financial interests that relate to the research described in this paper.

${ }^{\dagger}$ Go to http://dx.doi.org/10.1257/aer.20140662 to visit the article page for additional materials and author disclosure statement(s).
} 
gains to trade are small relative to the underlying information asymmetry-rent seeking via socially inefficient screening reduces the expected surplus from trade.

Yet we show that involving a moderately informed intermediary, whose information quality ranks between the buyer's and the seller's, can improve efficiency. By layering trade over two sequential transactions rather than one, this form of intermediation may better incentivize efficient trading behavior by all agents involved. The benefits of a moderately informed intermediary further extend to settings where several intermediaries trade an asset sequentially, as part of an intermediation chain in which each trader's information set is similar, although not identical, to those of his direct counterparties. Thus, in contrast to other theories that highlight the benefits of a single intermediary, our analysis provides a rationale for why assets may be traded through long intermediation chains rather than through simpler networks centered around one dominant broker.

Below we provide a simple example aimed at illustrating the intuition behind our main results.

Illustrative Example.-The monopolist seller of an asset (or good) must choose the price he will quote to a potential buyer (or customer) as a take-it-or-leave-it offer. The seller is, however, uncertain about how much the buyer is willing to pay for the asset. In particular, the seller only knows that the buyer's valuation of the asset, which we denote by $v$, has a uniform distribution: $v \sim U[1,2]$. The buyer only accepts to pay a price $p$ if $v \geq p$; otherwise, the seller must retain the asset, which for now, is assumed to be worth a constant value $c<1$ to him. The seller's optimization problem can then be summarized as follows:

$$
\max _{p \in[1,2]} \Pi(p)=\operatorname{Pr}(v \geq p) p+\operatorname{Pr}(v<p) c=(2-p) p+(p-1) c .
$$

Concavity of $\Pi(\cdot)$ implies that when $\Pi^{\prime}(1)=c \leq 0$, the seller quotes a price $p=1$, which is always accepted by the buyer. Thus, trade occurs with probability 1 if and only if $c \leq 0$, which is the socially efficient outcome since the buyer always values the asset more than the seller. However, if the seller's valuation of the asset is $c>0$, the seller finds it optimal to quote $p>1$ and use his market power to screen the privately informed buyer, inefficiently destroying gains to trade with positive probability.

Now suppose that we involve an intermediary who, like the seller, only values the asset at $c$. Given this private valuation, the intermediary only helps realize gains to trade if he resells the asset to the buyer. What may facilitate allocative efficiency is the fact that this intermediary is assumed to be moderately informed, in the sense that he obtains a signal indicating whether $v \in[1,1.5)$ or $v \in[1.5,2]$, that is, the signal pools either above-median or below-median realizations of the buyer's valuation of the asset. The intermediary is thus better informed than the original seller, but less informed than the buyer. The seller first quotes a price to the intermediary. If the intermediary accepts to pay this price and obtains the asset he then quotes a price to the buyer. Using backward induction, we show that trade may now be efficient even when $c>0$. 
Conditional on holding the asset and knowing that $v<1.5$, the intermediary's optimization problem when quoting a price to the buyer is

(2) $\max _{p \in[1,1.5)} \operatorname{Pr}(v \geq p \mid v<1.5) p+\operatorname{Pr}(v<p \mid v<1.5) c=\left(\frac{1.5-p}{0.5}\right) p+\left(\frac{p-1}{0.5}\right) c$.

The first-order condition of this problem implies that the seller quotes a socially efficient price $p=1$ if and only if $c \leq 0.5$. Similarly, conditional on holding the asset and knowing that $v \geq 1.5$, the intermediary's optimization problem can be written as

(3) $\max _{p \in[1.5,2]} \operatorname{Pr}(v \geq p \mid v \geq 1.5) p+\operatorname{Pr}(v<p \mid v \geq 1.5) c=\left(\frac{2-p}{0.5}\right) p+\left(\frac{p-1.5}{0.5}\right) c$.

Here, the first-order condition implies that the seller quotes a socially efficient price $p=1.5$ if and only if $c \leq 1$. Overall, the intermediary thus always quotes a socially efficient price as long as $c \leq \min \{0.5,1\}=0.5$.

Now, consider the seller's problem provided that this condition is satisfied $(c \leq 0.5)$ and the seller anticipates that trade between the intermediary and the buyer will be efficient. Facing the intermediary, the seller then optimally chooses between quoting a price $p=1$, which is always accepted, and a price $p=1.5$, which the intermediary only accepts if he receives a signal that $v \geq 1.5$. Thus, the seller chooses to quote a price $p=1$ rather than $p=1.5$ if and only if

$$
1 \geq 0.5 \cdot 1.5+0.5 \cdot c
$$

which simplifies to $c \leq 0.5$. Together, these conditions imply that intermediated trade is efficient as long as $c \leq 0.5$, whereas direct trade can only be efficient if $c \leq 0$.

It is helpful to interpret this result by considering the trade-off between the probability of a sale and the payoff the seller collects conditional on a sale. The more dispersed the seller's beliefs about the buyer's valuation are, the less the seller reduces the probability of trade when increasing his price quote. A seller with more dispersed beliefs is thus more tempted to screen the buyer by quoting higher prices, while probabilistically destroying gains to trade. In the example above, without an intermediary the seller reduces the probability of trade by $\varepsilon$ when inefficiently increasing his price quote by $\varepsilon$. In contrast, the intermediary finds it less profitable to screen the buyer-given his better information and more concentrated beliefs, the intermediary reduces the probability of trade by $2 \varepsilon$ when inefficiently increasing his price quote by $\varepsilon$. Similarly, the original seller may find deviating from a socially efficient price less attractive when trading with the intermediary rather than directly with the buyer. Facing the intermediary, the seller's beliefs about his counterparty's valuation are concentrated at two points, corresponding to the two possible signal realizations that pool ranges of buyer types. A seller quoting a price that exceeds the intermediary's valuation of the asset given a low signal, even just marginally, is then penalized by a large decline in the probability of trade. Overall, this simple example highlights how replacing one monopoly problem with a large information asymmetry by two sequential monopoly problems, each with less information asymmetry, may reduce incentives to screen counterparties, thereby increasing the social efficiency of trade. 
We explore these results in greater detail throughout the paper by generalizing our analysis along several dimensions. We allow for traders' private information to relate to common and private value components. We also generalize our distributional assumptions on $v$ and how the intermediary's information sets partition the distribution. Finally, we show how involving multiple intermediaries can further improve the efficiency of trade. In the example above, we can use similar arguments to show that the condition for efficient trade is weakened to $c \leq 0.67$ if trade instead goes through a chain of two intermediaries: a first one who knows whether $v \in[1,1.67)$ or $v \in[1.67,2]$ and a second one who knows whether $v \in[1,1.33)$, $v \in[1.33,1.67)$, or $v \in[1.67,2]$.

When market power leads to inefficiencies, one might expect that adding layers of intermediation would reduce efficiency due to problems of double marginalization (e.g., Spengler 1950). Our paper, however, shows that if intermediaries are partially informed, the associated reduction in the incentives to screen throughout the intermediation chain can, somewhat paradoxically, improve efficiency. Moreover, if private information is sufficiently dispersed, longer chains of intermediaries may be needed to sustain efficient trade in each transaction. Greater information asymmetries thus require longer intermediation chains that involve many sequential transactions, contrasting with the conventional wisdom that asymmetric information should be associated with low trading volume (as it is the case in the seminal model of Akerlof 1970).

Related Literature.-Intermediation is known to facilitate trade, either by minimizing transaction costs (Townsend 1978), by concentrating monitoring incentives (Diamond 1984), or by alleviating search frictions (Rubinstein and Wolinsky 1987; Yavaş 1994; Duffie, Gârleanu, and Pedersen 2005; Neklyudov 2013). Our paper specifically speaks to how intermediaries may alleviate trading inefficiencies caused by asymmetric information and imperfect competition. We know from Myerson and Satterthwaite (1983) that an uninformed third party who subsidizes transactions can help eliminate problems of asymmetric information in bilateral trade. Trade efficiency can also be improved by the involvement of fully informed middlemen who care about their reputation (Biglaiser 1993) or who worry that informed buyers could force them to hold on to low-quality goods (Li 1998). Contrary to these models, our setup considers the possibility that an intermediary's information set differs from those of the other agents already involved in the transaction. In fact, in our static model without subsidies, warranties, or reputational concerns the involvement of an intermediary who is equally informed as either the buyer or the seller does not improve trade efficiency. Thus, the insight that involving moderately informed intermediaries can help solve a standard problem in economics - when an agent uses his market power to inefficiently screen his trade partner-fundamentally differentiates our paper from these earlier papers.

Although intermediation chains can be observed in various settings (e.g., retail distribution networks), we rely on the recent empirical literature documenting the importance of interdealer trading and intermediation chains in over-the-counter (OTC) financial markets to contextualize our theory. According to the Bank for International Settlements (2013), interdealer trading accounts for 35 percent of the $\$ 2.3$ trillion in daily transaction volume for OTC interest-rate derivatives. Goldstein 
and Hotchkiss (2012) find that roughly one-third of transaction volume in secondary markets for newly issued corporate bonds is among dealers. For municipal bonds, Li and Schürhoff (2014) show that 13 percent of intermediated round-trip trades involve a chain of 2 intermediaries and an additional 10 percent of trades involve 3 or more intermediaries. Hollifield, Neklyudov, and Spatt (2014) also provide evidence of intermediation chains in the market for securitized products: for example, intermediated round-trip trades of non-agency collateralized mortgage obligations (CMO) involve 1.76 dealers on average and in some instances the chain includes up to 10 dealers.

Viswanathan and Wang (2004) propose an alternative explanation for the existence of intermediation chains in OTC markets. ${ }^{1}$ In their model, a security issuer may prefer a trading venue where dealers with heterogeneous inventory levels trade the security sequentially to a centralized auction that splits the supply of the security among the dealers. Traders find it optimal to share inventory risk among themselves and, as a result, they resell only a fraction of their acquired position to their respective counterparty in the chain. Although we agree that inventory risk concerns are economically important in financial markets, these concerns cannot explain the existence of the particular chains that Li and Schürhoff (2014) document. Li and Schürhoff (2014) identify an intermediation chain in the municipal bond market only when a dealer buys and then sells the same quantity of a security to another dealer. Intermediation chains that split orders among dealers in order to share inventory risk might still be prevalent in this market, but the specific chains that $\mathrm{Li}$ and Schürhoff (2014) document simply cannot be rationalized by interdealer trading aimed at dispersing inventory through a network.

Related findings also lend support to our information-based theory of intermediation chains. Intermediaries in our model are still averse to holding inventories (i.e., nonzero positions) since they are not the efficient holders of assets. Yet, information asymmetries may prevent them from offloading assets to potential buyers, consistent with evidence in Jiang and Sun (2015) suggesting that the corporate bond market is affected by significant asymmetric information problems. Consistent with the mechanism at play in our model, Li and Schürhoff (2014) show that municipal bonds without credit ratings or with speculative ratings are typically traded through longer intermediation chains than municipal bonds with investment-grade ratings, which arguably are less likely to be associated with large information asymmetries. Further, Di Maggio, Kermani, and Song (2015) find that average chain length in the corporate bond market increased following Lehman Brothers' collapse, a time during which uncertainty and the potential for information asymmetries spiked.

More broadly, our paper can shed light on the observation by Adrian and Shin (2010, p. 604) that the whole US financial system shifted in recent decades from its traditional, centralized model of financial intermediation to a more complex, market-based model characterized by "the long chain of financial intermediaries

\footnotetext{
${ }^{1}$ In contrast, Weller (2013) documents high-frequency trading chains in metals futures markets and proposes a rationale for these particular chains that relies on heterogeneity in dealers' technological ability to quickly transact in centralized trading venues. Evaluating the effects of high-frequency trading, Jovanovic and Menkveld (2015) also consider a model with centralized trading but focus on the idea that limit orders can be adversely selected once new information arrives. They show theoretically and empirically that this type of adverse selection can be alleviated by the entry of high-frequency traders who refresh quotes frequently.
} 
involved in channeling funds" (see also for similar characterizations, Kroszner and Melick 2009; Cetorelli, Mandel, and Mollineaux 2012; Pozsar et al. 2013).

Roadmap.- - In the next section, we model a fairly standard bargaining problem between two asymmetrically informed traders. We analyze in Section II how involving a moderately informed intermediary can improve trade efficiency. In Section III, we extend our analysis to show how long chains of intermediaries may sustain efficient trade in cases where shorter chains do not. In Section IV, we discuss the implementation of this type of intermediation, and the last section concludes. Unless stated otherwise, proofs are relegated to the Appendix.

\section{Direct Bilateral Trade}

We initially consider two risk-neutral agents who wish to trade one unit of an asset over the counter: a potential buyer who values the asset ex post at $v \in\left[v_{L}, v_{H}\right]$ and the current owner of the asset who values it at $c(v)$. The function $c(\cdot)$ is weakly increasing, continuous, and satisfies $c(v)<v$ for all $v \in\left[v_{L}, v_{H}\right]$. The last condition implies that trade always creates a surplus and is therefore efficient if and only if the buyer obtains the asset with probability $1 .{ }^{2}$ The cumulative distribution function (CDF) for the value $v$, denoted by $F(v)$, is continuous and differentiable and the probability density function $(\mathrm{PDF})$, denoted by $f(v)$, takes strictly positive values everywhere on the support $\left[v_{L}, v_{H}\right]$. The functions $c(\cdot)$ and $F(\cdot)$ are common knowledge, but traders are asymmetrically informed about $v$ at the time of trade.

Although the role that intermediation plays in our model is relatively simple, multilayered bargaining problems with asymmetric information are usually complex and give rise to multiple equilibria. We therefore make a few stylized assumptions that will allow us to keep the model tractable, even when we extend the analysis to multiple sequential transactions occurring among a large set of heterogeneously informed traders.

First, we assume that, in every transaction, the current holder of the asset makes an ultimatum offer to his counterparty. Focusing on ultimatum offers simplifies the analysis of equilibrium bidding strategies and is consistent with the characterization of interdealer trading in financial markets by Viswanathan and Wang (2004, p. 3) as "very quick interactions." Ultimatum offers are also consistent with how Duffie (2012, p. 2) describes the typical negotiation process in OTC markets and the notion that each OTC dealer tries to maintain "a reputation for standing firm on its original quotes."

Second, we assume that prior to trading, the seller is uninformed about the realization of $v$, whereas the buyer is an expert who knows $v$. Note that for many financial products endowing a "buyer" with private information rather than the "seller" is an unrestrictive assumption; for example, a firm could be viewed as the buyer of an insurance policy, or, alternatively, as the seller of a risk exposure. Moreover, as will become clear later, it is a trader's temptation to inefficiently screen his privately informed counterparty that moderately informed intermediaries may help eliminate.

\footnotetext{
${ }^{2}$ In the context of financial markets, these gains to trade may originate from heterogeneous liquidity or hedging needs across traders.
} 
This inefficient trading behavior could also arise under alternative information structures, for example, if the roles were reversed and an uninformed buyer made an ultimatum offer to a privately informed seller, or if both agents had their own private information about the asset. In an earlier draft available upon request (Glode and Opp 2015), we showed that our main results can survive in these alternative settings.

Third, agents know how well informed their counterparties are, that is, they know the distributions of other traders' information about $v$. Seppi (1990) lends support to this assumption arguing that agents knowing the identity of their trading counterparties is an important distinction between OTC trading and centralized/exchange trading. ${ }^{3}$

Finally, throughout the paper we also assume that in knife-edge cases where a trader is indifferent between two actions, he picks the one that maximizes social surplus.

Together, these assumptions imply that the proposer in our bargaining game does not possess any superior information when making an offer to his counterparty. As a result, signaling concerns do not arise and we obtain a unique subgame-perfect Nash equilibrium under direct trade. Moreover, our setting allows us to study a classic problem in economics where a monopolist seller inefficiently screens buyers based on their privately known types.

Analysis.-A subgame-perfect Nash equilibrium in the direct trade game consists of a price that the seller quotes and an acceptance rule for each possible buyer type $v$ that are mutual best responses in every subgame. If the seller quotes a price $p$, the buyer accepts to buy the asset whenever $v \geq p$, which occurs with probability $[1-F(p)]$. Otherwise, the seller must retain the asset, which he values at $c(v)$. We can write the seller's expected payoff when quoting a price $p$ as

$$
\Pi(p) \equiv[1-F(p)] p+F(p) E[c(v) \mid v<p]
$$

where $E$ denotes the expectation operator. The change in the expected payoff from marginally increasing the price $p$ is then given by

$$
\begin{aligned}
\Pi^{\prime}(p)= & {[1-F(p)]-f(p) p+f(p) E[c(v) \mid v<p] } \\
& +F(p) \frac{\partial}{\partial p} E[c(v) \mid v<p]
\end{aligned}
$$

which simplifies to

$$
\Pi^{\prime}(p)=[1-F(p)]-f(p)[p-c(p)] .
$$

The first term on the right-hand side of equation (7) represents the seller's expected benefit from collecting a higher price when the buyer accepts the offer. The second

\footnotetext{
${ }^{3}$ Morris and Shin (2012) relax the common-knowledge assumption in a bilateral trading setup similar to the one in this section and show how the resulting coordination problems can magnify the effect adverse selection has on trade efficiency.
} 
term represents the expected cost from reducing the probability of trade and jeopardizing trade surplus. The seller thus faces a trade-off between his payoff when a sale occurs and the probability of a sale occurring, which is greatly influenced by the nature of his uncertainty about the buyer's valuation. Ceteris paribus, greater belief dispersion, as reflected by lower values for the density $f(\cdot)$, implies that the seller finds it less costly to increase his price quote, strengthening his incentives to inefficiently screen the buyer.

It is useful to rewrite the seller's marginal profit of increasing the price $p$ as follows:

$$
\Pi^{\prime}(p)=[1-F(p)][1-H(p)]
$$

where we define the function

$$
H(v) \equiv\left[\frac{f(v)}{1-F(v)}\right][v-c(v)] \quad \text { for } v \in\left[v_{L}, v_{H}\right)
$$

to represent the ratio of the above-mentioned cost and benefit of marginally increasing the price. The seller finds it optimal to quote a higher, less efficient price than $p$ whenever $H(p)<1$. If on the other hand $H(p) \geq 1$, the benefit of collecting a higher price is dominated by the cost of reducing the acceptance probability.

We impose the following regularity condition on the function $H(\cdot)$ to guarantee that the marginal profit function $\Pi^{\prime}(\cdot)$ crosses zero from above at most in one point, which ensures that we obtain a unique subgame-perfect Nash equilibrium under direct trade:

ASSUMPTION 1: $H(v)$ is strictly increasing in $v$ for $v \in\left[v_{L}, v_{H}\right)$.

Assumption 1 is closely related to the definition of a strictly regular environment by Fuchs and Skrzypacz (2015) as well as a standard assumption in auction theory that bidders' virtual valuation functions are strictly increasing (Myerson 1981). Moreover, when the gains to trade are independent of $v$, that is, when $v-c(v)=\Delta>0$, Assumption 1 simplifies to imposing that the hazard rate function $h(v) \equiv f(v) /[1-F(v)]$ is strictly increasing. It is easy to verify that this last condition is satisfied by the uniform distribution and by a range of (truncated) parameterizations of the Normal distribution, the chi-squared distribution, and the gamma distribution, to name only a few.

Socially efficient trade requires that the seller quotes a price that is accepted by the buyer with probability 1 . Since $f(v)$ is strictly positive everywhere on the support $\left[v_{L}, v_{H}\right]$, the maximum price that maintains efficient trade is $p=v_{L}$. As a result, direct trade is efficient if and only if

$$
\Pi^{\prime}\left(v_{L}\right) \leq 0
$$

or equivalently

$$
H\left(v_{L}\right) \geq 1
$$


Efficient trade may thus be sustained in our setting as long as the gains to trade $[v-c(v)]$ are high and the seller's beliefs are sufficiently concentrated (i.e., the density $f(v)$ is high enough) at the lower bound of the support. In this paper we primarily characterize the condition(s) required for efficient trade, which allows us to isolate the benefits of intermediation, even when considering arbitrary numbers of intermediaries ( see Section III). ${ }^{4}$

\section{Intermediated Trade}

In this section, we consider the involvement of an intermediary who is moderately informed, in the sense that he receives an imperfect signal about $v$, making him better informed than the seller but less informed than the buyer. Specifically, we assume that the intermediary receives one of $N$ possible signal realizations, each associated with a conditional distribution $F_{i}(v)$ for $v$, where $i \in\{1,2, \ldots, N\}$. The probability of collecting each signal $i$ is denoted by $\pi_{i}>0$. We also assume that $F_{i+1}(\cdot)$ first-order stochastically dominates $F_{i}(\cdot)$ and we define the conditional upper and lower bounds $\quad \underline{v}_{i} \equiv \inf \left\{v \in\left[v_{L}, v_{H}\right]: F_{i}(v)>0\right\} \quad$ and $\quad \bar{v}_{i} \equiv \sup \left\{v \in\left[v_{L}, v_{H}\right]: F_{i}(v)<1\right\}$. Just like the seller, this intermediary privately values the asset at $c(v)$ and thus cannot help realize gains to trade unless he resells the asset to the buyer and thereby facilitates a more efficient allocation. Moreover, this intermediary does not bring new information to the table, as his information set is nested by that of the buyer. However, as we show below, intermediation by this moderately informed agent can improve the efficiency of trade. We consider a simple trading network in which the original seller can offer the asset only to the intermediary. If the intermediary purchases the asset he can offer to sell it to the buyer. As noted above, all offers are ultimatum offers. A subgame-perfect Nash equilibrium in this intermediated trade game consists of a sequence of prices and acceptance rules for all trader types that are mutual best responses in every subgame (including subgames that are off the equilibrium path).

To sustain efficient trade the intermediary has to quote prices that the buyer accepts with probability 1 . The intermediary must therefore find it optimal to quote $p=\underline{v}_{i}$ to the buyer after receiving signal $i$, since $\underline{v}_{i}$ represents the buyer's lowest possible valuation of the asset. A necessary condition for efficient trade is that a marginal deviation from this efficient price reduces the intermediary's conditional expected payoff, that is,

$$
\Pi_{i}^{\prime}\left(\underline{v}_{i}\right)=\left[1-F_{i}\left(\underline{v}_{i}\right)\right]\left[1-H_{i}\left(\underline{v}_{i}\right)\right] \leq 0 \quad \text { for } \quad i \in\{1,2, \ldots, N\},
$$

where we define $H_{i}(v) \equiv\left[\frac{f_{i}(v)}{1-F_{i}(v)}\right][v-c(v)]$ on the domain $v \in\left[\underline{v}_{i}, \bar{v}_{i}\right)$. Moreover, if the functions $H_{i}(v)$ are strictly increasing in $v$ on their respective domains, these $N$ inequalities become sufficient conditions for efficient trade between the intermediary and the buyer.

\footnotetext{
${ }^{4}$ See, e.g., d'Aspremont and Gérard-Varet (1979); Myerson and Satterthwaite (1983); and Samuelson (1984) for general analyses of the conditions required to implement first-best allocations under asymmetric information.
} 
Since the available surplus from trade $[v-c(v)]$ does not change with the involvement of an intermediary, it is the shape of the $N$ conditional distributions $F_{i}(\cdot)$ that dictates whether efficient trade can be sustained with an intermediary. If for some signal $i$ the conditional density $f_{i}(\cdot)$ is sufficiently small at the conditional lower bound $\underline{v}_{i}$, then marginally increasing the price has so little impact on the probability of trade that the intermediary finds it optimal to deviate from the socially efficient price $\underline{v}_{i} .{ }^{5}$ However, if the intermediary's signals concentrate posterior beliefs in specific regions of the unconditional support $\left[v_{L}, v_{H}\right]$ such that the conditional densities $f_{i}(\cdot)$ are sufficiently high at their respective lower bounds, then marginal deviations from the socially efficient price are privately suboptimal for the intermediary. As the intermediary gets to know the buyer's valuation with more precision, he has weaker incentives to inefficiently screen the buyer based on his private information and jeopardize gains to trade.

Efficient trade through the network also requires that the intermediary accepts the seller's offer with probability 1 . Since stochastic dominance implies that $\underline{v}_{1}=v_{L}$, the seller has to prefer quoting a price $p=v_{L}$ to the intermediary rather than higher prices that the intermediary accepts only after receiving any of the better signals $i \geq 2$. Provided that the conditions for efficient trade between the intermediary and the buyer are satisfied, the intermediary's conditional valuation for the asset is given by the price he will quote to the buyer: $\underline{v}_{i}$.

Overall, the involvement of an intermediary leads to the replacement of the condition for efficient trade under direct trade (i.e., equation (10)) by a set of $(N+1)$ conditions that depend on the shapes of the conditional distributions $F_{i}(\cdot)$. As we show below, partitions are a particular type of conditional distributions that strictly weakens the condition for efficient trade. In this regard, it is useful to establish the following Lemma (the proof is relegated to the online Appendix).

LEMMA 1: If Assumption 1 is satisfied under some distribution $F(\cdot)$, it is also satisfied under any truncated version of that distribution.

Assumption 1 thus guarantees that the intermediary's conditional marginal profit function $\Pi_{i}^{\prime}(\cdot)$ also crosses zero from above at most in one point. This property ensures that we obtain a unique subgame-perfect Nash equilibrium under intermediated trade. ${ }^{6}$ We can now establish our first main result.

PROPOSITION 1: Let $\Omega^{1}(F)$ and $\Omega^{0}(F)$ denote the set of functions $c(\cdot)$ associated with efficient trade with and without an intermediary, respectively, for a given CDF $F(\cdot)$, under Assumption 1. If the intermediary's signal partitions the support of $v$ into $N \geq 2$ subintervals of strictly positive measure, then the set of functions $c(\cdot)$ associated with efficient trade is strictly larger with the intermediary, that is, $\Omega^{0}(F) \subset \Omega^{1}(F)$.

\footnotetext{
${ }^{5}$ In particular, if the intermediary's conditional distributions have full support, then involving this intermediary cannot eliminate all trade inefficiencies since generically for some signals the intermediary's temptation to screen the buyer will be higher than the original seller's.

${ }^{6}$ As under direct trade, a strictly increasing $H_{i}(\cdot)$ function implies that the intermediary's optimal price quote to the buyer is unique. By backward induction, the seller can anticipate the intermediary's unique optimum response to any price quote, which implies a unique subgame-perfect Nash equilibrium (recall the tie-breaking rule we imposed for knife-edge cases where a trader is indifferent between two actions).
} 
Proposition 1 shows that the involvement of an intermediary with a specific signal structure can facilitate efficient trade: if the intermediary's information set partitions the interval $\left[v_{L}, v_{H}\right]$ into two or more subintervals, then intermediated trade is efficient for a strictly larger set of $c(\cdot)$ functions than direct trade. This result can be intuitively understood by considering the trade-off each potential holder of the asset faces when choosing a price to quote. When holding the asset, a trader's expected surplus from quoting a price to his better informed counterparty is the difference between the total expected surplus from trade and the information rents that can be appropriated by all subsequent traders in the network.

As under direct trade, each potential proposer trades off the negative impact of quoting a higher price on the probability of trade, and thus trade surplus, against the benefit from appropriating a larger share of the whole surplus if trade is realized. The impact of a price increase on the probability of trade is in turn affected by his counterparty's informational advantage, and therefore changes with the involvement of a moderately informed intermediary.

Since the intermediary is better informed about $v$ than the seller, the intermediary has lower incentives to inefficiently screen the buyer to reduce his information rents. In particular, as the intermediary's posterior beliefs are concentrated in a subinterval of the unconditional support $\left[v_{L}, v_{H}\right]$, the intermediary can quote prices above $v_{L}$ without jeopardizing any gains to trade (whenever $i \geq 2$ ). However, given that the intermediary's beliefs are concentrated in the $i$ th subinterval, any price increase beyond the conditional lower bound $\underline{v}_{i}$ is penalized by a larger decline in the conditional probability of trade. In the limiting case where the intermediary's partition becomes so fine that his information set is identical to the buyer's, a small increase above the maximum efficient price implies that the conditional probability of trade drops from one to zero, making such an inefficient deviation unattractive.

Further, provided that the intermediary trades efficiently with the buyer, the seller may also be more inclined to trade efficiently with the intermediary. Intuitively, screening is less effective when facing the moderately informed intermediary instead of the fully informed buyer. Under direct trade, the seller can screen the buyer by directly choosing the marginal buyer type from the continuous set $\left[v_{L}, v_{H}\right]$. In contrast, when facing an intermediary who plans to resell the asset at one of the lower bounds $\underline{v}_{i}$, the seller is effectively restricted to choosing the marginal buyer type from the discrete subset $\left\{v_{L}, \underline{v}_{2}, \ldots, \underline{v}_{N}\right\}$; the seller's beliefs about the intermediary's valuation of the asset are concentrated at these $N$ valuations. It follows immediately that if the seller optimally chooses the efficient price $v_{L}$ under direct trade he will also do so under intermediated trade, since the set of his alternative choices is weakly inferior when facing the intermediary. Moreover, we show in the proof of Proposition 1 the existence of a set of functions $c(\cdot)$ for which the seller chooses a marginally inefficient price, say $p^{\prime}>v_{L}$, under direct trade, but picks the efficient price $p=v_{L}$ when facing the intermediary and choosing from the set $\left\{v_{L}, \underline{v}_{2}, \ldots, \underline{v}_{N}\right\}$. Overshooting to a price of $\underline{v}_{2}$ is then inferior relative to $v_{L}$, as it brings about a significant reduction in the probability of trade. The larger the overshooting vis à vis $p^{\prime}$ - that is, the higher $\underline{v}_{2}$ - the less attractive is it for the seller to deviate from the efficient price $v_{L}$ when facing an intermediary.

Intermediation adds a strategic agent who uses his information and market power to capture a share of the trade surplus. Thus, as discussed above, the seller generally 
faces an inferior opportunity set when facing the intermediary, and therefore, would prefer to bypass this trader and contact the buyer directly. Yet Proposition 1 shows that there always exist valuation functions $c(\cdot)$ (that may depend on the type of assets being traded or the economic conditions at the time of the trade), for which this deviation would reduce the social efficiency of trade. A trading network achieving a higher social surplus then centers around a moderately informed intermediary and is sparse, in the sense that the seller cannot contact the buyer directly. In many decentralized markets, it is often impossible for retail or unsophisticated traders to contact the most sophisticated traders directly and bypass the usual middlemen. In fact, Li and Schürhoff (2014) estimate that for the municipal bond market only 2.4 percent of all possible directed links are formed, highlighting that sparsity is an empirically plausible feature of our model. Moreover, we highlight in Section IV that ex ante transfers, such as payments for order flow, can play a beneficial role in ensuring that the socially efficient trading network is implemented in equilibrium.

It is important to note that although involving the described intermediaries expands the set of functions $c(\cdot)$ for which efficient trade is sustained, it does not improve trade efficiency in all cases. When direct trade is inefficient the effect of intermediation generally depends on which buyer types are pooled by the intermediary's signals. Consider the involvement of an intermediary who can trade efficiently with the buyer and whose partition cutoff $\underline{v}_{2}$ is located just slightly above $p^{\prime}$, the price that maximizes the seller's expected payoff under direct trade. Facing the intermediary, the seller then might quote $p=\underline{v}_{2}$ since it is the price closest to $p^{\prime}$ within the set $\left\{v_{L}, \underline{v}_{2}, \ldots, \underline{v}_{N}\right\}$. However, since $\underline{v}_{2}>p^{\prime}$, the expected surplus from trade shrinks with the involvement of this intermediary. In contrast, if the intermediary's partition cutoff $\underline{v}_{2}$ is located slightly below $p^{\prime}$, then quoting $p=\underline{v}_{2}$ to the intermediary increases the expected surplus from trade relative to direct trade, making intermediation socially beneficial.

So far, our discussion focused on cases where the intermediary and the buyer trade efficiently. However, when trade is expected to break down with positive probability between the intermediary and the buyer, the original seller benefits less from trading efficiently with the intermediary. In these cases, intermediation not only affects the sensitivity of the probability of trade to a price increase, as illustrated above, but also the expected trade surplus at stake. Problems of double marginalization may then arise as two monopolists are sequentially trading the asset in an inefficient manner (Spengler 1950).

While involving intermediaries strictly expands the set of cases where efficient trade obtains, it may have ambiguous effects when trade remains inefficient. We show in the next section, however, that in those cases lengthening the chain by involving additional intermediaries may be sufficient to eliminate all inefficiencies.

\section{Intermediation Chains}

This section extends our earlier results and shows how long chains of intermediaries may sustain efficient trade in cases where shorter chains do not. In contrast to most models of intermediation where the optimal trading network is centered around a unique intermediary, our model sheds light on the prevalence of intermediation chains in many decentralized markets (Goldstein and Hotchkiss 2012; Bank 
for International Settlements 2013; Hollifield, Neklyudov, and Spatt 2014; Li and Schürhoff 2014; Di Maggio, Kermani, and Song 2015).

Suppose there are $M$ intermediaries, indexed by $m$ based on their position in a trading chain. To simplify the notation, we label the seller as trader 0 and the buyer as trader $M+1$. Each intermediary $m$ observes a signal that partitions the domain $\left[v_{L}, v_{H}\right]$ into subintervals. The mechanism that makes intermediation chains valuable in our model is an extension of that featured in Section II and is best highlighted by assuming that the information set intermediary $m$ has before trading is nested by the information set of intermediary $(m+1)$. That is, intermediary $(m+1)$ observes a signal that creates a strictly finer conditional partition than intermediary $m$ 's signal. Formally, we make the following assumption:

ASSUMPTION 2: If intermediary $m<M$ knows that $v \in\left[\underline{v}_{i}^{m}, \bar{v}_{i}^{m}\right)$, then intermediary $(m+1)^{\prime} s$ information partitions $\left[\underline{v}_{i}^{m}, \bar{v}_{i}^{m}\right)$ into at least three subintervals of strictly positive measure.

Nesting sequential traders' information sets in this fashion eliminates signaling concerns and implies a unique subgame-perfect Nash equilibrium in our model, despite the presence of $(M+1)$ bargaining problems among $(M+2)$ heterogeneously informed agents. In this game with multiple intermediaries, the definition of a subgame-perfect Nash equilibrium is the natural counterpart of the definition stated in Section II. Assuming that there are at least three subintervals that separate each pair of counterparties $m$ and $(m+1)$ guarantees that we are able to insert a "moderately informed" intermediary between them, if needed. This particular structure will allow us to extend some of our earlier results and show that long intermediation chains can preserve the efficiency of trade in situations where surplus would be destroyed with fewer intermediaries. What ultimately contributes to sustaining efficient trade in equilibrium is that the chain reduces the informational distance between counterparties, although information sets would not necessarily have to be nested for our mechanism to work. 7

The proposition below formalizes our main result regarding intermediation chains.

PROPOSITION 2: Let $\Omega^{M}(F)$ denote the set of functions $c(\cdot)$ associated with efficient trade in a chain of $M$ intermediaries with information sets satisfying Assumption 2 for a given $C D F F(\cdot)$, under Assumption 1. There exists a set of $\tilde{M} \geq 1$ intermediaries who can be added to the chain such that the set of functions $c(\cdot)$ associated with efficient trade is strictly enlarged, that is, $\Omega^{M}(F) \subset \Omega^{M+\tilde{M}}(F)$.

As before, when holding the asset a proposer's expected payoff from quoting a price is the difference between the total surplus from trade and the cumulative information rents going to all subsequent traders in the chain. Each proposer trades off the negative impact of quoting a higher price on the probability of trade, and thus trade surplus, against the benefit from appropriating a larger share of the whole surplus

\footnotetext{
${ }^{7}$ In an earlier draft (Glode and Opp 2015), available upon request, we studied variants of our model with non-nested information sets and obtained qualitatively similar results.
} 
if trade is realized. If the responder has only a small informational advantage over the proposer, deviating from efficient trade by quoting aggressive prices imposes large reductions in the probability of trade. Yet, provided that trade is efficient in subsequent transactions, the surplus from trade that would be destroyed in each transaction by quoting aggressive prices is the same. When anticipating efficient trade in later transactions, strategies aimed at quoting inefficient prices to counterparties are thus discouraged in a long intermediation chain. Thus, by locating traders within a chain such that each trader's information set is similar, but not identical, to those of his direct counterparties efficient trade can be facilitated. For example, in the context of OTC markets the least sophisticated dealer should trade directly with uninformed retail investors while the most sophisticated dealer should trade directly with the best hedge funds and trading desks on Wall Street. In a nonfinancial context, our results highlight the potential benefits of multilayered distribution channels where local retailers quote prices to privately informed customers. Proposition 2 also implies that, if the functions $c(\cdot)$ and $F(\cdot)$ are perturbed in ways that worsen the efficiency of trade in a given chain, a higher number of intermediaries may be needed to bridge the information asymmetries and sustain efficient trade, consistent with the empirical evidence from Li and Schürhoff (2014) and Di Maggio, Kermani, and Song (2015) discussed in our introduction.

Our results also highlight that the optimality of a trading network greatly depends on the trading frictions that are most relevant in a given context. When trade is impeded by inefficient screening of privately informed counterparties, our model shows that multiple heterogeneously informed intermediaries may improve the social efficiency of trade. When private information relates to traders' past behavior instead, Babus (2012) shows that optimal trading networks should be centered around a single intermediary who penalizes anyone defaulting on prior obligations (see also Farboodi 2014). Further, Gofman (2011) shows that in the presence of noninformational bargaining frictions, socially efficient outcomes may be easier to achieve when networks are dense (although the relationship between density and efficiency is not always monotonic)..$^{8}$ In contrast, in our model a trading network needs to be sufficiently sparse to sustain efficient trade; otherwise, less informed parties have incentives to directly contact highly informed traders and inefficiently screen them. We discuss in the next section the role that payments for order flow can play in alleviating problems of this type. Given that some trading frictions are more relevant in some situations than in others, our results and those derived in the papers mentioned above can help us understand the types of networks we observe in different contexts.

Note also that the decentralized market structure we study is empirically prevalent and contrasts with alternative interventions that promote greater competition by centralizing traders' interactions. In particular, if multiple informed traders were to bid simultaneously for the seller's asset and a Crémer and McLean (1988)-type mechanism was allowed, the seller could use competition to effectively extract information from bidders. This competition effect is, however, absent in our setting where trade is bilateral and the asset moves through each trader sequentially. The

\footnotetext{
${ }^{8}$ See also Wright and Wong (2014) who study the impact of non-informational bargaining and search frictions on trade in chains of intermediaries.
} 
seller does not extract any information from competing bidders, but rather faces a single intermediary who is less informed than the ultimate buyer. This smaller information gap between the seller and his counterparty strengthens the seller's incentives to quote an efficient price. Further, as highlighted by Biais, Martimort, and Rochet (2000), while moving from a monopolistic to an oligopolistic market structure may reduce incentives to inefficiently screen privately informed agents in the presence of adverse selection, it generally does not completely eliminate these inefficiencies. Hence, equilibrium trading volume under oligopolistic screening is below its socially optimal level, just as in the monopoly case. The intermediation chains we study feature decentralized, sequential trading among heterogeneously informed agents and are thus different from these alternative mechanisms.

\section{Implementation}

So far, we have shown that intermediated trade can improve trade efficiency when traders have incentives to inefficiently screen their privately informed counterparties. In this section, we consider a network-formation game that precedes the trading game from Section III. We characterize order-flow agreements that traders commit to ex ante, that is, before information is obtained and trading occurs. These order-flow agreements ensure that no trader involved in an intermediation chain that sustains efficient trade will be tempted to form an alternative trading network. Order-flow agreements can thus help implement socially optimal trading networks in our model, shedding light on potential downsides of recent proposals by regulators and stock exchange officials to ban related practices in financial markets.?

DEFINITION 1: Consider an economy with a set of traders $\mathcal{T}$. An order-flow agreement $\Sigma$ between a subset of traders $\mathcal{S} \subseteq \mathcal{T}$ specifies the following objects:

(i) A collection of directed network links: each trader $m \in \mathcal{S}$ is exclusively connected to a unique counterparty $m^{\prime} \in\{\mathcal{S} \backslash m\}$ to which trader $m$ quotes an ultimatum price whenever he wishes to sell.

(ii) A collection of ex ante transfers between the traders in $\mathcal{S}$.

A key component of these order-flow agreements are ex ante transfers that incentivize traders to commit to transacting with specific counterparties. In financial markets, these transfers may come in the form of explicit agreements involving cash payments for order flow or soft dollars, or they may be implicit arrangements involving profitable IPO allocations or subsidies on the various other services that intermediaries provide. In fact, there is ample empirical evidence that "perks" are commonly used by financial intermediaries to compensate traders for

\footnotetext{
${ }^{9}$ See, for example, the comments made by Jeffrey Sprecher (CEO of Intercontinental Exchange, which owns the New York Stock Exchange), reported in 'ICE CEO Sprecher Wants Regulators to Look at 'Maker-Taker' Trading" by Christine Stebbins (Reuters.com, January 26, 2014); "Guidance on the Practice of 'Payment for Order Flow," prepared by the Financial Services Authority (May 2012); and the comments made by Harvey Pitt (former Securities and Exchange Commission Chairman), reported in "Options Payment for Order Flow Ripped" by Isabelle Clary (Securities Technology Monitor, May 3, 2004).
} 
their business (see, e.g., Blume 1993; Chordia and Subrahmanyam 1995; Reuter 2006; Nimalendran, Ritter, and Zhang 2007). Further, for many types of securities, order-flow agreements are required to be disclosed in advance in Rule 606 reports. Thus, just like in our definition above, transfers linked to order-flow agreements do not vary based on transaction-specific information (i.e., a particular realization of $v$ ), but they may vary based on the expertise of the traders involved (as documented by Easley, Kiefer, and O'Hara 1996). This characterization distinguishes these ex ante transfers from the transfers that occur ex post when transaction prices are paid as part of the trading process.

In the network-formation game we consider, each trader's payoff is the sum of these ex ante transfers and continuation payoffs that are consistent with the equilibrium outcome(s) of the trading game described in Section III. We define an equilibrium of this network-formation game as follows.

DEFINITION 2: An order-flow agreement $\Sigma$ between a set of traders $\mathcal{S} \subseteq \mathcal{T}$ constitutes an equilibrium of the network-formation game if there is no coalition of traders $\mathcal{S}^{\prime} \subseteq \mathcal{T}$ that can block the agreement, that is, there does not exist an order-flow agreement $\Sigma^{\prime}$ that only includes traders in $\mathcal{S}^{\prime}$ and that makes every trader in $\mathcal{S}^{\prime}$ weakly better off and at least one trader in $\mathcal{S}^{\prime}$ strictly better off.

Consistent with our previous analysis, we are interested in the cases for which intermediation chains help sustain efficient trade. As noted above, networks satisfying Assumption 2 have a unique equilibrium in the trading game and thus unique continuation payoffs for all traders. Below we characterize the existence of equilibrium order-flow agreements that support the type of intermediation chains we introduced in Section III (the proof is relegated to the online Appendix).

PROPOSITION 3: If the set $\mathcal{T}$ contains traders endowed with information sets consistent with Assumption 2 who can form a chain that would sustain efficient trade in the trading game:

(i) Any order-flow agreement that would not sustain efficient trade is not an equilibrium in the network-formation game.

(ii) For any intermediation chain that would sustain efficient trade, there exists a corresponding order-flow agreement that constitutes an equilibrium in the network-formation game.

The result in Proposition 3 relies on the following logic. Suppose there exists an equilibrium in which the trading network is such that the equilibrium of the subsequent trading game is inefficient. Then, agents can create a new coalition that includes all traders that can sustain efficient trade and offer ex ante transfers to all agents that were in the original network while also collecting the additional surplus that is available when trade is efficient. This set of agents thus constitutes a blocking coalition, a contradiction.

In our model, deal-flow is valuable to intermediaries because they extract information rents and thus obtain a fraction of the total surplus from trade $E[v-c(v)]$. 
Intermediaries are willing to offer cash payments, or subsidized services, to the ultimate buyer and seller of the asset if these are required concessions for being involved in the trading network.

\section{Conclusion}

This paper shows that chains of moderately informed intermediaries may alleviate trading inefficiencies associated with the screening of privately informed counterparties by traders with market power. Layering trade over multiple sequential transactions that involve moderately informed intermediaries can weaken each trader's incentives to screen counterparties, and thereby increase the efficiency of trade. Greater information asymmetries may thus be better bridged by long intermediation chains in which each trader's information set is similar, although not identical, to those of his direct counterparties. We note, however, that in cases where efficient trade is not achievable despite the presence of intermediaries, intermediation may sometimes make trade more fragile as problems of double marginalization can arise. A full characterization of the environments where heterogeneously informed intermediaries either improve or worsen the efficiency of trade in markets with private information is left for future research.

\section{APPENDIX}

\section{PROOF OF PROPOSITION 1:}

We will first show that $\Omega^{0}(F) \subseteq \Omega^{1}(F)$. Let $\Psi(F)$ denote the set of functions $c(\cdot)$ consistent with the regularity condition stated in Assumption 1 for a given CDF $F(\cdot)$. We can thus characterize the set $\Omega^{0}(F)$ as

$$
\Omega^{0}(F)=\left\{c \in \Psi(F): \Pi^{\prime}\left(v_{L}\right) \leq 0\right\} .
$$

Trade between the Intermediary and the Buyer-To show that $c \in \Omega^{0}(F) \Rightarrow c \in \Omega^{1}(F)$, we first show that the intermediary, when holding the asset, quotes a price that the buyer accepts with probability 1 , provided that $c \in \Omega^{0}(F)$. If the intermediary receives a signal $i$ that implies that $v \in\left[\underline{v}_{i}, \bar{v}_{i}\right)$, he chooses a price $p$ to maximize his conditional expected payoff:

$$
\Pi_{i}(p)=\left[1-F_{i}(p)\right] p+F_{i}(p) E[c(v) \mid v<p, i] .
$$

By Lemma $1, H_{i}(v)$ is strictly increasing on $\left[\underline{v}_{i}, \bar{v}_{i}\right)$ and a sufficient condition for efficient trade between the intermediary and the buyer is

$$
\Pi_{i}^{\prime}\left(\underline{v}_{i}\right)=\left[1-F_{i}\left(\underline{v}_{i}\right)\right]\left[1-H_{i}\left(\underline{v}_{i}\right)\right] \leq 0 .
$$

By definition, for all $c \in \Omega^{0}(F)$ we know that $\Pi^{\prime}\left(v_{L}\right) \leq 0$, or equivalently, $H\left(v_{L}\right) \geq 1$. We now show that $H_{i}\left(\underline{v}_{i}\right)>H\left(v_{L}\right)$ for all $i$, which allows us to conclude that the intermediary quotes a socially efficient price, since $H\left(v_{L}\right) \geq 1$ then implies that $H_{i}\left(\underline{v}_{i}\right)>1$ and equivalently, $\Pi_{i}^{\prime}\left(\underline{v}_{i}\right)<0$, for all $i \in\{1, \ldots, N\}$. 
When $\underline{v}_{i}>v_{L}$ we obtain the inequality

$$
\begin{aligned}
H_{i}\left(\underline{v}_{i}\right) & =\left[\frac{f\left(\underline{v}_{i}\right)}{F\left(\bar{v}_{i}\right)-F\left(\underline{v}_{i}\right)}\right]\left[\underline{v}_{i}-c\left(\underline{v}_{i}\right)\right] \\
& \geq\left[\frac{f\left(\underline{v}_{i}\right)}{1-F\left(\underline{v}_{i}\right)}\right]\left[\underline{v}_{i}-c\left(\underline{v}_{i}\right)\right]=H\left(\underline{v}_{i}\right)>H\left(v_{L}\right),
\end{aligned}
$$

and when $\bar{v}_{i}<v_{H}$, we obtain

$$
\begin{aligned}
H_{i}\left(\underline{v}_{i}\right) & =\left[\frac{f\left(\underline{v}_{i}\right)}{F\left(\bar{v}_{i}\right)-F\left(\underline{v}_{i}\right)}\right]\left[\underline{v}_{i}-c\left(\underline{v}_{i}\right)\right] \\
& >\left[\frac{f\left(\underline{v}_{i}\right)}{1-F\left(\underline{v}_{i}\right)}\right]\left[\underline{v}_{i}-c\left(\underline{v}_{i}\right)\right]=H\left(\underline{v}_{i}\right) \geq H\left(v_{L}\right) .
\end{aligned}
$$

Trade between the Seller and the Intermediary.-Next, we show that the seller quotes the intermediary a price of $v_{L}$ rather than any of the price candidates $\underline{v}_{i}$ for $i \in\{2,3, \ldots, N+1\}$, where we define $\underline{v}_{N+1} \equiv v_{H}$. We can restrict our attention to this discrete set of price candidates, since any prices lying between these price candidates are strictly dominated: these intermediate prices imply the same probability of trade as the next-higher price in the set, but a lower sales price. Thus, provided that the conditions for efficient trade between the intermediary and the buyer are satisfied, a sufficient condition for efficient trade between the seller and the intermediary is

(A6) $v_{L} \geq\left[1-F\left(\underline{v}_{i}\right)\right] \underline{v}_{i}+F\left(\underline{v}_{i}\right) E\left[c(v) \mid v<\underline{v}_{i}\right] \quad$ for $\quad i \in\{2, \ldots, N+1\}$.

At the cutoffs $\underline{v}_{i}$, we can use the function $\Pi(p)$ introduced in equation $(5)$ and rewrite equation (A6) as

$$
\Pi\left(\underline{v}_{i}\right)-\Pi\left(v_{L}\right) \leq 0 \text { for } i \in\{2, \ldots, N+1\} .
$$

This representation shows that, when facing the intermediary, the seller's expected payoff is weakly lower than under direct trade: the discrete set of attainable expected payoffs $\left\{\Pi\left(v_{L}\right), \Pi\left(\underline{v}_{2}\right), \ldots, \Pi\left(v_{H}\right)\right\}$ is a strict subset of the continuous set of expected payoffs attainable under direct trade, $\{\Pi(v)\}_{v \in\left[v_{L}, v_{H}\right]}$. It follows immediately that if the seller does not want to deviate from the efficient price $v_{L}$ under direct trade he will not wish to do so under intermediated trade as well. To summarize, since we have shown that both the intermediary and the seller trade efficiently under intermediated trade whenever direct trade is efficient, it follows that $\Omega^{0}(F) \subseteq \Omega^{1}(F)$.

It remains to be shown that $\Omega^{0}(F) \subset \Omega^{1}(F)$, that is, there exist functions $c(\cdot)$ such that $c \in \Omega^{1}(F)$ but $c \notin \Omega^{0}(F)$. We can write the seller's expected payoff from deviating from the socially efficient price as follows:

$$
\Pi\left(\underline{v}_{i}\right)-\Pi\left(v_{L}\right)=\int_{v_{L}}^{\underline{v}_{i}} \Pi^{\prime}(z) d z .
$$


Since $\Pi^{\prime}(\cdot)$ crosses zero from above at most in one point on the support $\left[v_{L}, v_{H}\right]$, it follows that $\Pi^{\prime}\left(v_{L}\right) \leq 0$ implies that $\Pi\left(\underline{v}_{i}\right)-\Pi\left(v_{L}\right)<0$ for all $i \geq 2$. That is, when facing the intermediary, deviating from the efficient price makes the seller strictly worse off. The same property of $\Pi^{\prime}(\cdot)$ implies that if $\Pi\left(\underline{v}_{2}\right)-\Pi\left(v_{L}\right) \leq 0$ then $\Pi\left(\underline{v}_{i}\right)-\Pi\left(v_{L}\right) \leq 0$ for all $i \geq 2$. We can therefore characterize the set $\Omega^{1}(F)$ as follows:

$$
\Omega^{1}(F)=\left\{c \in \Psi(F): \Pi\left(\underline{v}_{2}\right)-\Pi\left(v_{L}\right) \leq 0,\left\{\Pi_{i}^{\prime}\left(\underline{v}_{i}\right) \leq 0\right\}_{\forall i \in N}\right\}
$$

By continuity of the set $\Psi(F)$, there exists a subset of functions $c \in \Omega^{0}(F)$ for which the condition for efficient direct trade holds with equality, that is, where $\Pi^{\prime}\left(v_{L}\right)=0$. Consider replacing any one of these functions $c(v)$ by a perturbed function $\tilde{c}(v, \epsilon) \equiv c(v)+\epsilon$, where $\epsilon \geq 0$ is bounded from above to ensure that $\tilde{c}(v, \epsilon)$ still satisfies the regularity condition $v-\tilde{c}(v, \epsilon)>0$ for all $v \in\left[v_{L}, v_{H}\right]$. Define the overall slack across all efficiency conditions in the presence of the intermediary under such a function $\tilde{c}(v, \epsilon)$ as

$$
\tilde{\rho}(\epsilon) \equiv-\max \left\{\Pi\left(\underline{v}_{2}\right)-\Pi\left(v_{L}\right),\left\{\Pi_{i}^{\prime}\left(\underline{v}_{i}\right)\right\}_{\forall i \in N}\right\}
$$

Note that all $\Pi_{i}^{\prime}\left(\underline{v}_{i}\right)$ are continuous and strictly increasing in $\epsilon$ and so is the difference $\Pi\left(\underline{v}_{2}\right)-\Pi\left(v_{L}\right)$. Moreover, applying the maximum operator to a set of continuous and strictly increasing functions yields a continuous and strictly increasing function. Thus, $\tilde{\rho}(\epsilon)$ is continuous and strictly decreasing in $\epsilon$.

We showed above that when the condition for efficient direct trade holds with equality $\left(\Pi^{\prime}\left(v_{L}\right)=0\right)$ then, after introducing an intermediary, all conditions for efficient trade hold with strict inequality, i.e., $\tilde{\rho}(0)>0$. By continuity of $\tilde{\rho}(\epsilon)$ there exist strictly positive values for $\varepsilon$ such that $\tilde{\rho}(\epsilon) \geq 0$, implying that $\tilde{c}(v, \epsilon) \in \Omega^{1}(F)$. Yet, since we started with a function $c(v)=\tilde{c}(v, 0)$ for which $\Pi^{\prime}\left(v_{L}\right)=0$, these perturbed functions $\tilde{c}(v, \epsilon)$ will imply that $\Pi^{\prime}\left(v_{L}\right)>0$ such that $\tilde{c}(v, \epsilon) \notin \Omega^{0}(F)$. It thus follows that the set of functions $c(v)$ that satisfy efficient trade with the intermediary is strictly larger than the one without the intermediary, that is, $\Omega^{0}(F) \subset \Omega^{1}(F)$.

\section{PROOF OF PROPOSITION 2:}

The logic of this proof is similar to the one of Proposition 1. Yet the notation is more involved as we need to keep track of multiple layers of intermediation. In the following we will use the subscript $m$ to identify trader $m$ 's information set. For example, $F_{m}(v)$ is the CDF of $v$ given trader $m$ 's information set, $E_{m}[v]$ is the expectation of $v$ given trader $m$ 's information set, and

$$
\Pi_{m}(p) \equiv\left[1-F_{m}(p)\right] p+F_{m}(p) E_{m}[c(v) \mid v<p]
$$

If trader $m<M$ knows that $v \in\left[\underline{v}_{i}^{m}, \bar{v}_{i}^{m}\right)$ then trader $(m+1)$ knows that $v$ is in one of the $K(m+1, i) \geq 3$ non-overlapping subintervals associated with the boundaries $w_{j}(m+1, i)$, where $\underline{v}_{i}^{m}=w_{0}(m+1, i)$ $<w_{1}(m+1, i)<\cdots<w_{K}(m+1, i)=\bar{v}_{i}^{m}$. Thus, $w_{j}(m+1, i)$ denotes the $j$ th partition cutoff of trader $(m+1)$ 's information set if trader $m$ observes signal $i \in N_{m}$. For a given $F(\cdot)$ and a given chain with $M$ intermediaries we define $\Omega^{M}(F)$ 
as the set of functions $c(\cdot)$ that satisfy all conditions for efficient trade along the chain:

$$
\begin{aligned}
\Omega^{M}(F)= & \left\{c \in \Psi(F):\left\{\Pi_{M}^{\prime}\left(\underline{\underline{M}}_{i}^{M}\right) \leq 0\right\}_{\forall i \in N_{M},}\right. \\
& \left.\left\{\Pi_{m}\left(w_{1}(m+1, i)\right)-\Pi_{m}\left(w_{0}(m+1, i)\right) \leq 0\right\}_{\forall i \in N_{m}} \forall m<M\right\},
\end{aligned}
$$

where $\Psi(F)$ is defined as in the proof of Proposition 1. Also as in the proof of Proposition 1 , in any transaction between non-experts $(m<M)$, we can focus on verifying the profitability of a trader $m$ 's deviation from a marginal type $w_{0}(m+1, i)$ to the type $w_{1}(m+1, i)$ due to the fact that $\Pi_{m}^{\prime}(v)$ crosses zero from above at most once (in one point).

By continuity of the set $\Psi(F)$, there exist functions $c \in \Omega^{M}(F)$ such that for some transaction between traders $m$ and $(m+1)$ the condition for efficient trade holds with equality after some signal $i$, that is, either:

$$
\Pi_{m}\left(w_{1}(m+1, i)\right)-\Pi_{m}\left(w_{0}(m+1, i)\right)=0 \quad \text { if } \quad m<M
$$

or

$$
\Pi_{M}^{\prime}\left(\underline{\underline{x}}_{i}^{M}\right)=0 \quad \text { if } \quad m=M .
$$

We will show next that the conditions for efficient trade hold with strict inequality if we introduce a particular intermediary $\tilde{m}$ between these two traders $m$ and $(m+1)$. This intermediary $\tilde{m}$ knows that $v$ is in one of $\tilde{K} \in\{2, \ldots, K(m+1, i)-1\}$ non-overlapping subintervals associated with the boundaries $\tilde{w}_{i}$, where $\underline{v}_{i}^{m}=w_{0}(m+1, i)=\tilde{w}_{0}<\tilde{w}_{1}<\cdots<\tilde{w}_{\tilde{K}}=w_{K}(m+1, i)$ $=\bar{v}_{i}^{m}$. Moreover, if $m<M$, these boundaries are assumed to satisfy $\tilde{w}_{i} \in\left\{w_{2}(m+1, i), \ldots, w_{K}(m+1, i)\right\}$ for all $i \in\{1,2, \ldots, \tilde{K}\}$. Trader $\tilde{m}$ 's partition is thus a strict refinement of trader $m$ 's partition, and trader $(m+1)$ 's partition is a strict refinement of trader $\tilde{m}$ 's partition. Going forward, we analyze a generic transaction in the chain characterized by the tuple $(i, m)$, and, for notational simplicity, will simply write $w_{j}$ when referring to $w_{j}(m+1, i)$.

Trade between Intermediary $\tilde{m}$ and Trader $(m+1)$ if $m<M$.-Trader $\tilde{m}$ observes that $v \in\left[\tilde{w}_{j}, \tilde{w}_{j+1}\right)$, where $j \in\{0,1, \ldots, \tilde{K}-1\}$. First, consider the case where the intermediary $\tilde{m}$ 's signal implies that $\tilde{w}_{j}>w_{0}$. Analogously to arguments in the proof of Proposition $1, \Pi_{m}\left(w_{1}\right)-\Pi_{m}\left(w_{0}\right)=0$ implies that $\Pi_{m}^{\prime}\left(w_{1}\right)<0$ and equivalently, $H_{m}\left(w_{1}\right)>1$. Further, since $H_{\tilde{m}}(v) \geq H_{m}(v)$ it follows that $H_{\tilde{m}}(v)>1$ for all $v>w_{1}$. Thus, when $\tilde{w}_{j} \geq w_{1}$, the condition for efficient trade between traders $\tilde{m}$ and $(m+1)$ holds with strict inequality since

$$
\Pi_{\tilde{m}}\left(w_{k}\right)-\Pi_{\tilde{m}}\left(\tilde{w}_{j}\right)=\int_{\tilde{w}_{j}}^{w_{k}}\left[1-F_{\tilde{m}}(z)\right]\left[1-H_{\tilde{m}}(z)\right] d z<0,
$$


where we define $k=\min \left\{s \in\{1,2, \ldots, K(m+1, i)\}: w_{s}>\tilde{w}_{j}\right\}$. As for the case where $\tilde{w}_{j}=w_{0}$, we can write

$$
\begin{aligned}
\Pi_{\tilde{m}}\left(w_{1}\right)-\Pi_{\tilde{m}}\left(w_{0}\right) & =\int_{w_{0}}^{w_{1}}\left[1-F_{\tilde{m}}(z)\right]\left[1-H_{\tilde{m}}(z)\right] d z \\
& =\int_{w_{0}}^{w_{1}}\left[\frac{F_{m}\left(\tilde{w}_{1}\right)-F_{m}(z)}{F_{m}\left(\tilde{w}_{1}\right)-F_{m}\left(w_{0}\right)}\right]\left[1-\left(\frac{f_{m}(z)}{F_{m}\left(\tilde{w}_{1}\right)-F_{m}(z)}\right)(z-c(z))\right] d z \\
& =\frac{\int_{w_{0}}^{w_{1}}\left[1-F_{m}(z)\right]\left[\frac{F_{m}\left(\tilde{w}_{1}\right)-F_{m}(z)}{1-F_{m}(z)}-\left(\frac{f_{m}(z)}{1-F_{m}(z)}\right)(z-c(z))\right] d z}{F_{m}\left(\tilde{w}_{1}\right)-F_{m}\left(w_{0}\right)} \\
& =\frac{\int_{w_{0}}^{w_{1}}\left[1-F_{m}(z)\right]\left[1-H_{m}(z)-\frac{1-F_{m}\left(\tilde{w}_{1}\right)}{1-F_{m}(z)}\right] d z}{F_{m}\left(\tilde{w}_{1}\right)-F_{m}\left(w_{0}\right)} \\
& =\frac{\Pi_{m}\left(w_{1}\right)-\Pi_{m}\left(w_{0}\right)-\int_{w_{0}}^{w_{1}}\left[1-F_{m}(z)\right]\left[\frac{1-F_{m}\left(\tilde{w}_{1}\right)}{1-F_{m}(z)}\right] d z}{F_{m}\left(\tilde{w}_{1}\right)-F_{m}\left(w_{0}\right)}
\end{aligned}
$$

which means that $\Pi_{m}\left(w_{1}\right)-\Pi_{m}\left(w_{0}\right)=0 \Rightarrow \Pi_{\tilde{m}}\left(w_{k}\right)-\Pi_{\tilde{m}}\left(\tilde{w}_{0}\right)<0$, since

$$
-\frac{\int_{w_{0}}^{w_{1}}\left[1-F_{m}(z)\right]\left[\frac{1-F_{m}\left(\tilde{w}_{1}\right)}{1-F_{m}(z)}\right] d z}{F_{m}\left(\tilde{w}_{1}\right)-F_{m}\left(w_{0}\right)}<0 .
$$

Trade between Intermediary $\tilde{m}$ and Trader $(m+1)$ if $m=M$.-When trader $(m+1)$ is the expert buyer $(M+1)$, the condition for efficient trade is given by

$$
\Pi_{\tilde{m}}^{\prime}\left(\tilde{w}_{j}\right)=\left[1-F_{\tilde{m}}\left(\tilde{w}_{j}\right)\right]\left[1-H_{\tilde{m}}\left(\tilde{w}_{j}\right)\right] \leq 0 \quad \forall j \geq 0
$$

We want to show that this condition is satisfied with strict inequality whenever $\Pi_{m}^{\prime}\left(w_{0}\right)=0$. In that case, we know that $H_{m}\left(w_{0}\right)=1$, which, according to the derivations above, implies that $H_{\tilde{m}}\left(w_{0}\right)>1$ and $H_{\tilde{m}}\left(\tilde{w}_{j}\right)>1$ for all $j \geq 0$. Thus, $\Pi_{m}^{\prime}\left(w_{0}\right)=0 \Rightarrow \Pi_{\tilde{m}}^{\prime}\left(\tilde{w}_{j}\right)<0$ for all $j \geq 0$.

Trade between Trader $m$ and Intermediary $\tilde{m}$.- Trader $m$ quotes a socially efficient price to trader $\tilde{m}$ when

$$
\Pi_{m}\left(\tilde{w}_{1}\right)-\Pi_{m}\left(w_{0}\right)<0 .
$$

If $m<M$, that is, trader $(m+1)$ is not the expert buyer, then we know that $\tilde{w}_{1}>w_{1}$ and we can rewrite this condition as

$$
\Pi_{m}\left(w_{1}\right)-\Pi_{m}\left(w_{0}\right)+\int_{w_{1}}^{\tilde{w}_{1}} \Pi_{m}^{\prime}(z) d z<0 .
$$


If trader $(m+1)$ is the expert buyer and $\Pi_{m}^{\prime}\left(w_{0}\right)=0$, then we also know that $\Pi_{m}^{\prime}(w)<0$ for $w>w_{0}$, and thus $\Pi_{m}\left(\tilde{w}_{1}\right)-\Pi_{m}\left(w_{0}\right)<0$.

Adding $\tilde{M} \geq 1$ Intermediaries to the Chain.- Suppose that for a given function $c(\cdot)$ and a chain with $M$ intermediaries, there are $\tilde{M} \operatorname{transaction}(\mathrm{s})$ in the chain where a condition for efficient trade holds with equality (for at least one possible signal). The derivations above imply that introducing $\tilde{M}$ new traders, with information sets that satisfy the conditions described above, to intermediate these $\tilde{M}$ transactions ensures that all conditions in the chain with $(M+\tilde{M})$ intermediaries hold with strict inequality. Now, consider replacing any one of these functions $c(v)$ by a perturbed function $\tilde{c}(v, \epsilon) \equiv c(v)+\epsilon$, where $\varepsilon \geq 0$ is bounded from above to ensure that $v-\tilde{c}(v, \epsilon)>0$ for all $v \in\left[v_{L}, v_{H}\right]$. We can define the overall slack across all efficiency conditions in the new chain with $(M+\tilde{M})$ intermediaries under the function $\tilde{c}(v, \epsilon)$ as $\tilde{\rho}(\epsilon)$ and use the same steps as in the proof of Proposition 1 to show that $\Omega^{M}(F) \subset \Omega^{M+\tilde{M}}(F)$.

\section{REFERENCES}

-Adrian, Tobias, and Hyun Song Shin. 2010. "The Changing Nature of Financial Intermediation and the Financial Crisis of 2007-2009.” Annual Review of Economics 2: 603-18.

-Akerlof, George A. 1970. "The Market for 'Lemons': Quality Uncertainty and the Market Mechanism." Quarterly Journal of Economics 84 (3): 488-500.

Babus, Ana. 2012. "Endogenous Intermediation in Over-the-Counter Markets." http://ssrn.com/ abstract $=2630156$.

Bank for International Settlements. 2013. The OTC Interest Rate Derivatives Market in 2013. Basel, Switzerland: International Banking and Financial Market Developments.

- Biais, Bruno, David Martimort, and Jean-Charles Rochet. 2000. "Competing Mechanisms in a Common Value Environment." Econometrica 68 (4): 799-837.

- Biglaiser, Gary. 1993. "Middlemen as Experts." RAND Journal of Economics 24 (2): 212-23.

- Blume, Marshall E. 1993. "Soft Dollars and the Brokerage Industry." Financial Analysts Journal 49 (2): 36-44.

Cetorelli, Nicola, Benjamin H. Mandel, and Lindsay Mollineaux. 2012. "The Evolution of Banks and Financial Intermediation: Framing the Analysis.” FRBNY Economic Policy Review (July): 1-12.

Chordia, Tarun, and Avanidhar Subrahmanyam. 1995. "Market Making, the Tick Size, and Paymentfor-Order Flow: Theory and Evidence.” Journal of Business 68 (4): 543-75.

-Crémer, Jacques, and Richard P. McLean. 1988. "Full Extraction of the Surplus in Bayesian and Dominant Strategy Auctions." Econometrica 56 (6): 1247-57.

-d'Aspremont, Claude, and Louis-André Gérard-Varet. 1979. "Incentives and Incomplete Information." Journal of Public Economics 11 (1): 25-45.

Diamond, Douglas W. 1984. "Financial Intermediation and Delegated Monitoring." Review of Economic Studies 51 (3): 393-414.

Di Maggio, Marco, Amir Kermani, and Zhaogang Song. 2015. "The Value of Trading Relationships in Turbulent Times." http://ssrn.com/abstract=2597938.

Duffie, Darrell. 2012. Dark Markets: Asset Pricing and Information Transmission in Over-the-Counter Markets. Princeton: Princeton University Press.

-Duffie, Darrell, Nicolae Gârleanu, and Lasse Heje Pedersen. 2005. "Over-the-Counter Markets." Econometrica 73 (6): 1815-47.

-Easley, David, Nicholas M. Kiefer, and Maureen O'Hara. 1996. "Cream-Skimming or Profit-Sharing? The Curious Role of Purchased Order Flow." Journal of Finance 51 (3): 811-33.

Farboodi, Maryam. 2014. "Intermediation and Voluntary Exposure to Counterparty Risk." http://ssrn. com/abstract $=2535900$.

Fuchs, William, and Andrzej Skrzypacz. 2015. "Government Interventions in a Dynamic Market with Adverse Selection.” Journal of Economic Theory 158 (Part A): 371-406.

Glode, Vincent, and Christian Opp. 2015. "Adverse Selection and Intermediation Chains." Unpublished. 
Gofman, Michael. 2011. “A Network-Based Analysis of Over-the-Counter Markets.” http://ssrn.com/ abstract $=1681151$.

Goldstein, Michael A., and Edith S. Hotchkiss. 2012. "Dealer Behavior and the Trading of Newly Issued Corporate Bonds.” http://ssrn.com/abstract=1022356.

Hollifield, Burton, Artem Neklyudov, and Chester Spatt. 2014. "Bid-Ask Spreads and the Pricing of Securitizations: 144a vs. Registered Securitizations.” http://ssrn.com/abstract=2493244.

Jiang, Hao, and Zheng Sun. 2015. "News and Corporate Bond Liquidity." http://ssrn.com/ abstract $=2437975$.

Jovanovic, Boyan, and Albert J. Menkveld. 2015. "Middlemen in Limit Order Markets.” http://ssrn. com/abstract $=1624329$.

Kroszner, Randall S., and William Melick. 2009. "The Response of the Federal Reserve to the Recent Banking and Financial Crisis." Unpublished.

Li, Dan, and Norman Schürhoff. 2014. "Dealer Networks.” http://ssrn.com/abstract=2023201.

Li, Yiting. 1998. "Middlemen and Private Information." Journal of Monetary Economics 42 (1): 131-59.

Morris, Stephen, and Hyun Song Shin. 2012. "Contagious Adverse Selection.” American Economic Journal: Macroeconomics 4 (1): 1-21.

Myerson, Roger B. 1981. “Optimal Auction Design.” Mathematics of Operations Research 6 (1): 58-73.

Myerson, Roger B., and Mark A. Satterthwaite. 1983. "Efficient Mechanisms for Bilateral Trading." Journal of Economic Theory 29 (2): 265-81.

Neklyudov, Artem V. 2013. "Bid-Ask Spreads and the Decentralized Interdealer Markets: Core and Peripheral Dealers." Unpublished.

Nimalendran, M., Jay R. Ritter, and Donghang Zhang. 2007. “Do Today's Trades Affect Tomorrow's IPO Allocations?” Journal of Financial Economics 84 (1): 87-109.

Pozsar, Zoltan, Tobias Adrian, Adam Ashcraft, and Hayley Boesky. 2013. "Shadow Banking." FRBNY Economic Policy Review (December): 1-16.

Reuter, Jonathan. 2006. "Are IPO Allocations for Sale? Evidence from Mutual Funds." Journal of Finance 61 (5): 2289-2324.

Rubinstein, Ariel, and Asher Wolinsky. 1987. "Middlemen.” Quarterly Journal of Economics 102 (3): 581-94.

-Samuelson, William. 1984. "Bargaining under Asymmetric Information.” Econometrica 52 (4): 995-1006.

-Seppi, Duane J. 1990. "Equilibrium Block Trading and Asymmetric Information.” Journal of Finance 45 (1): 73-94.

-Spengler, Joseph. 1950. "Vertical Integration and Antitrust Policy." Journal of Political Economy $58(4): 347-52$.

Townsend, Robert M. 1978. "Intermediation with Costly Bilateral Exchange." Review of Economic Studies 45 (3): 417-25.

Viswanathan, S., and James J. D. Wang. 2004. "Inter-Dealer Trading in Financial Markets." Journal of Business 77 (4): 1-54.

Weller, Brian. 2013. "Liquidity and High Frequency Trading." Unpublished.

Wright, Randall, and Yuet-Yee Wong. 2014. "Buyers, Sellers, and Middlemen: Variations on Search-Theoretic Themes." International Economic Review 55 (2): 375-97.

- Yavaş, Abdullah. 1994. "Middlemen in Bilateral Search Markets." Journal of Labor Economics 12 (3): 406-29. 